\title{
Controllo interno e di gestione nella prospettiva del valore
}

\author{
Aldo Pavan *
}

\begin{abstract}
Internal control and management control in the value creation perspective.

Each economic entity is aimed at generating value and durability. Various control tools are prepared. The management control continuously verifies the results achieved in comparison with the budget objectives. The internal control system and the enterprise risk management oversee the conditions for the achievement of the corporate objectives. The above control systems are often considered as separate entities. It is a paradox; both preside over the orientation towards value, objectives and efficiency. A strong commitment to the homogenization of control instruments is therefore necessary. Starting from this consideration, the VII Workshop of the magazine proposed, in Cagliari on 24 and 25 May 2018, the theme "Management control and internal control: value creation and stakeholders' protection". The presented papers dealt with the suggested themes, but they ranged on the various topics of interest to the magazine. The present issue presents seven selected articles that deal with internal control and managerial control, the definition of scenarios in strategic planning, the management rationale in non-profit foundations and the use of social media in Italian museums.
\end{abstract}

Keywords: internal control and management control, CoSo Report.

\section{Introduzione}

Il sistema d'azienda svolge l'attività di produzione ed è volto alla generazione di valore per i diversi portatori di interesse. Data una determinata missione, i vertici aziendali formulano gli obiettivi generali e particolari di ogni singola unità organizzativa, di breve e lungo periodo. La vita dell'organismo economico che operi quale entità autonoma nel mercato, in particolare, è condotta e valutata in base al principio di economicità aziendale perché esso possa durare nel tempo e costituire fattore di benessere per le comunità di riferimento. Si tratta di un equilibrio generale e durevole che può essere articolato in obiettivi elementari corrispondenti, da un lato, all'efficacia e all'efficienza nel raggiungimento degli obiettivi aziendali e, dall'altro

\footnotetext{
* Prof. Aldo Pavan, Dipartimento di scienze economiche e aziendali, Università degli studi di Cagliari, e-mail: apavan@unica.it.
}

Management Control (ISSN 2239-0391, ISSNe 2239-4397), 2019, 1 - Special Issue DOI: 10.3280/MACO2019-SU1001 
lato, agli aspetti reddituale, patrimoniale e monetario della gestione (Onida, 1965). L'efficacia è definita quale grado di raggiungimento degli obiettivi e l'efficienza in termini di razionale utilizzo delle risorse a disposizione nel perseguimento degli obiettivi medesimi.

La relazione tra costi e ricavi è vista nel senso della massimizzazione del valore economico dell'impresa. Quella tra fonti di finanziamento e fabbisogni finanziari è intesa quale adeguatezza dei primi rispetto ai secondi, da un lato, del patrimonio netto e delle altre fonti a non breve scadenza rispetto all'attivo immobilizzato e all'indebitamento corrente, dall'altro lato. L'armonia fra i flussi di disponibilità liquide infine deriva dai precedenti equilibri strutturali: reddituale e patrimoniale, e dalla generazione interna di cassa. L'equilibrio monetario si concretizza nella capacità di far fronte, nel breve periodo, alle passività correnti con le disponibilità in essere e le entrate correnti; prima ancora, nella capacità di assumere decisioni di spesa nella prospettiva del valore, piuttosto che sotto l'assillo di impellenti ristrettezze di cassa (Pavan e Fadda, 2017, pp. 36-37; Sòstero, 2003).

Definita la gestione, in senso soggettivo, quale insieme di decisioni, si individua un percorso ove le scelte sono precedute dall'approntamento di un adeguato supporto di conoscenza e seguite da coerenti atti di esecuzione. Il controllo scaturisce infine dal confronto sistematico e continuativo tra quanto era stato previsto nel budget d'esercizio e quanto scaturisce dalla rilevazione consuntiva. L'analisi degli scostamenti consente ai soggetti decisori di avere informazioni e tenere alta l'attenzione circa il processo di generazione del valore e il mantenimento durevole delle condizioni di economicità. Prende forma così il concetto di controllo di gestione, tradizionalmente orientato verso la redditività di breve periodo, i processi di gestione interna e le quantità di natura economica e finanziaria - Management Accounting System, MAS (Chiucchi e Gatti, 2015). Tale concetto evolve, nella teoria e nella prassi, verso il controllo definito "strategico" quando l'attenzione viene spostata verso la generazione durevole di valore, l'interazione dinamica con l'ambiente e le cause che danno luogo a determinate quantità economiche $\mathrm{e}$ finanziarie. In tale direzione vengono attuate azioni di benchmarking, con le quali i prodotti e processi aziendali sono posti a confronto con quelli dei concorrenti più agguerriti al fine di porre in essere prassi di miglioramento continuo. Si parla, per contro, di balanced scorecard quando vengono definite misure che consentano di tenere sotto controllo non solo la dimensione finanziaria di generazione del valore, ma anche altre in qualche modo "causali" di questa. Si tratta più spesso della soddisfazione dei clienti, dalla quale derivano i ricavi di vendita; dell'adeguatezza dei processi, che determina il 
livello dei costi e la qualità dei prodotti; della crescita professionale che pone l'attenzione sulla professionalità e la motivazione delle risorse umane.

Da un altro punto di vista, il controllo è inteso quale sistema di strumenti volti, in positivo, al presidio delle condizioni e delle vie gestionali verso gli obiettivi aziendali e, in negativo, al contrasto di fenomeni di cattiva gestione e corruzione. Il sistema di controllo interno - SCI - è definito dal documento, emanato nel 1992 e poi rivisto sino all'ultima edizione del 2013, dal Committee of Sponsoring organizations of the Treadway Commission e comunemente noto come CoSo Report I. Tale documento è oggi considerato il riferimento per eccellenza per ogni tipo di aziende pubbliche e private. Esso può esser opportunamente letto alla luce del concetto di "scienza del controllo economico", formulato nel 1880 da Fabio Besta quale essenza della ragioneria Besta, (1880). Secondo il Chiaro Autore, il controllo economico, o riscontro, è volto alla tutela del patrimonio aziendale, a impedire sottrazioni colpevoli e inutili consumi e a rendere più efficace il lavoro di direzione. Esso comprende le registrature dei fatti amministrativi e le misure di controllo. Sono condizioni per il controllo: la sapiente distribuzione di organismi amministrativi e di funzioni, la selezione del personale, i sistemi incentivanti. Nell'ultima versione della sua La Ragioneria (1922), al capitolo XXX è sviluppato il concetto di "costrizione degli atti amministrativi" reso concreto da strumenti del controllo concomitante quali: la vigilanza, la naturale opposizione di interessi tra soggetti, l'utilizzo di documenti e congegni automatici (Landis et al., 2012). La vigilanza è basata sull'indipendenza del vigilante ed è svolta con ispezioni impreviste, ma attese. La naturale opposizione di interessi presuppone che nessuna operazione o negozio può essere portato a termine da una sola persona. È suggerito l'utilizzo di strumenti materiali quali documenti di carico e scarico, sigilli e chiavi, congegni automatici al tempo disponibili, quali contatori a funzionamento meccanico. Nel pensiero del Besta e dei suoi allievi sono rinvenibili elementi attuali del controllo quali: ambiente e attività di controllo, informazione e comunicazione, efficacia ed efficienza, tutela del patrimonio. Rispetto allo sviluppo attuale della materia, risultano invece assenti i temi dell'affidabilità del bilancio, della conformità a leggi e regolamenti, della valutazione dei rischi e del monitoraggio continuo dell'adeguatezza degli strumenti di controllo.

Il CoSo Report I definisce il controllo interno quale processo svolto dal $\mathrm{CdA}$, dai dirigenti e da altro personale incaricato di fornire una ragionevole sicurezza circa il raggiungimento degli obiettivi aziendali. Lo SCI ha degli obiettivi suoi propri, declinati in: a) efficacia ed efficienza delle operazioni di gestione, compresa la salvaguardia del patrimonio; b) affidabilità della 
rendicontazione contabile; c) conformità quale rispetto delle leggi e dei regolamenti. Strumentali a tali obiettivi sono cinque componenti; due di tipo strutturale: ambiente di controllo, informazione e comunicazione; tre dinamici: valutazione del rischio, attività di controllo e monitoraggio. L'ambiente di controllo attiene alla deontologia e alla professionalità e può pertanto essere declinato nei valori etici condivisi, con particolare riferimento all'esempio dei capi - il tono al vertice -, nell'adeguatezza dell'organizzazione, nella competenza professionale, nella gestione e incentivazione del personale. L'informazione e comunicazione attiene all'efficacia e all'efficienza del sistema informativo, così che le informazioni siano utili, tempestive, complete, chiare e riservate, inoltre disponibili ove servono e in formato adeguato alle necessità dell'utente. La valutazione del rischio considera la gestionale e la salvaguardia del patrimonio, l'attività di rendicontazione e la conformità ed è sviluppata partendo dalla definizione degli obiettivi generali e derivati, per poi continuare con l'identificazione, valutazione e gestione dei rischi interni ed esterni e concludersi con l'individuazione di meccanismi, volti ad appropriate modificazioni nella gestione in risposta cambiamenti dannosi. Le attività di controllo costituiscono l'aspetto materiale del processo e si concretizzano in strumenti diversi quali, a titolo esemplificativo, la revisioni da parte dell'alta dirigenza, i controlli sul sistema informativo, la segregazione dei compiti, la rotazione delle mansioni, le diverse procedure di autorizzazione e approvazione, i controlli fisici. Il monitoraggio costituisce infine il punto di raccordo tra il controllo, quale attività in qualche modo interna alla gestione attraverso la quale il controllo si autoregola, e la revisione interna. Esso verifica l'adeguatezza nel tempo del SCI - per es. nuovi rischi -, attraverso attività continue in quanto integrate nei processi e valutazioni specifiche dell'efficacia dell'intero sistema.

Nel 2002 viene emanato il CoSo Report II - aggiornato da ultimo nel 2017 - che ha per oggetto l'Enterprise Risk Management - ERM. Si tratta di uno standard di per sé autonomo rispetto allo SCI, ma che questo comprende e sviluppa ponendo enfasi sui concetti di individuazione e gestione dei rischi. Rispetto al CoSo I tra gli obiettivi è aggiunta la prospettiva strategica, mentre la valutazione del rischio è preceduta da altri due componenti: la definizione degli obiettivi e l'identificazione degli eventi rischiosi. L'ERM è definito quale processo, effettuato dal consiglio di amministrazione, dall'alta direzione e da altro personale aziendale, svolto nella definizione della strategia e con riferimento a tutta l'impresa, progettato per identificare potenziali eventi che possono influire sull'impresa stessa e per gestire i rischi all'interno della propria propensione al rischio, al fine di assicurare una ragionevole garanzia in merito al raggiungimento degli obiettivi aziendali (D’Onza, 2008). 
Le due dimensioni del controllo che precedono sono spesso considerate, nella letteratura e nella pratica, quali entità a sé stanti (Arena e Arnaboldi 2014; Soin e Collier, 2013). Si tratta a evidenza di un paradosso quando si adotti un approccio sistemico all'amministrazione economica d'azienda. Si tratta comunque di presidiare l'orientamento al valore del complesso aziendale, l'orientamento agli obiettivi e all'efficienza, l'equilibrio nei diversi aspetti della gestione, il presidio degli elementi causali dai quali il valore durevole è generato, la correttezza dell'informazione interna ed esterna e il rispetto di leggi e regolamenti. Occorre d'altro canto contrastare, ma meglio prevenire, fenomeni di corruzione e cattiva gestione, di "sottrazioni colpevoli e inutili consumi", attraverso la tempestiva individuazione dei molteplici rischi e la loro opportuna gestione. Appare dunque necessario un forte impegno verso l'omogeneizzazione degli strumenti di controllo.

Partendo da tale considerazione il VII Workshop della rivista ha proposto, a Cagliari il 24 e 25 maggio del 2018, il tema "Controlli interni e di gestione: generazione di valore e tutela dei diversi portatori di interesse. Le relazioni presentate hanno trattato $i$ temi specifici suggeriti, ma hanno spaziato sui diversi temi di interesse della rivista. Il presente volume presenta sette lavori selezionati e giunti tempestivamente a compimento; altri, meritevoli di pubblicazione, saranno pubblicati in numeri successivi.

\section{Il contenuto del fascicolo}

L'articolo di Cristiana Cattaneo e Gaia Bassani tratta, in modo specifico, del percorso di integrazione tra ERM e MAS in un'importante banca di credito cooperativo. L'ambiente bancario, fortemente regolato, impone l'utilizzo dei diversi strumenti di controllo e consente la sperimentazione di forme di integrazione. Sono indagati, in modo particolare, le relazioni che influenzano i processi decisionali e i fattori che rendono efficace l'integrazione medesima. Rileva in primo luogo la presenza di responsabili di processi che guidano l'integrazione e il convinto coinvolgimento dell'alta direzione. È osservata poi la presenza di un sistema informativo progettato e realizzato in funzione delle esigenze degli utenti. Un ruolo particolare è svolto infine dalla diffusione di una cultura aziendale in cui tutti i membri dell'organizzazione sono consapevoli del significato intrinseco delle informazioni per le decisioni e dei rischi correlati. Tali fattori sembrano favorire la simbiosi tra ERM e MAS, così da stimolare il reale interesse degli utenti e far sì che i dirigenti utilizzino le informazioni integrate per il processo decisionale quotidiano. 
Selena Aureli, Andrea Cardoni, Mara Del Baldo e Rosa Lombardi si occupano di reti aziendali tra piccole e medie imprese - PMI - e si chiedono, in particolare, se gli strumenti tradizionali di controllo di gestione contribuiscono a promuovere il dialogo e l'innovazione tra le entità componenti. È osservato un caso di studio, costituito da una rete fondata su rapporti contrattuali e operante, in Italia, nel settore della carta e della stampa. Il contesto orizzontale, piuttosto che gerarchico, della rete presenta delle opportunità particolari di utilizzo innovativo dei pur tradizionali strumenti di MAS, alla condizione di adattare al caso specifico la distinzione tra controlli diagnostici e interattivi proposta da Simons negli anni '90. I primi sono volti a limitare la creatività delle risorse umane al solo sviluppo di nuovi prodotti, i secondi si propongono al contrario di stimolare la propensione all'innovazione, grazie all'utilizzo interattivo delle misure di risultato. L'utilizzo interattivo degli strumenti di controllo nelle reti può essere dedicato allo stimolo delle idee e alla condivisione delle conoscenze al fine di formare una cultura di collaborazione professionale e manageriale.

Enzo Bivona indaga il fenomeno delle infiltrazioni criminali nelle imprese e pone il quesito sul se e come il SCI può costituire un presidio efficace di prevenzione e, se del caso, di individuazione e bonifica contro le azioni della malavita organizzata. Il tema è rilevante soprattutto per gli appaltatori di opere pubbliche chiamati a verificare l'affidabilità di partner e fornitori con i quali interagire. L'assenza di efficaci strumenti di prevenzione e contrastato dei tentativi di infiltrazioni della criminalità organizzata nella gestione aziendale può dar luogo infatti a sanzioni interdittive in grado di compromettere in modo irreversibile l'immagine aziendale e il processo di creazione di valore e tutela degli stakeholder. L'indagine è condotta su un caso di studio, nello specifico costituita da un'impresa, operante nel Nord Italia, interessata a tale tipo di situazioni nonostante fosse dotata di un modello di organizzazione e gestione ex D.Lgs. n. 231/01. Nella logica dell'action research, l'implementazione del SCI ha consentito la soluzione di una situazione compromessa in modo significativo.

Con Anna Maria Arcari, Mara Del Baldo e Marcantonio Ruisi torniamo al tema del controllo di gestione, con particolare riferimento alle PMI. Gli autori indagano il tipo e il livello di diffusione degli strumenti in argomento adottando la prospettiva di professionisti esterni, dottori commercialisti ed esperti contabili. Attraverso un questionario, è stato chiesto ai professionisti facenti parte di un campione selezionato nel Centro-Sud d'Italia quanto essi siano chiamati sostenere gli imprenditori nell'implementazione del controllo. I risultati evidenziano un approccio professionale incentrato principalmente sulla fornitura di servizi in tema di contabilità e adempimenti tributari, 
nonostante la consapevolezza della necessità di promuovere una più ampia azione di consulenza che includa l'adozione di adeguati strumenti di controllo di gestione. Emerge la prospettiva di una figura professionale specializzata che possa affiancare l'imprenditore nella transizione verso la prospettiva strategica e manageriale.

Filippo Zanin ed Eugenio Comuzzi propongono il primo di tre lavori che spaziano su temi più ampi. Qui è trattato il ruolo di supporto della metodologia alla definizione degli scenari nelle attività di pianificazione strategica. Il tema è controverso e necessita di una sistemazione tanto teorica quanto orientata all'implementazione. Gli autori si sono mossi su due direttrici, dapprima l'analisi della letteratura ha permesso di identificare quattro temi centrali ricorrenti: il ruolo della pianificazione degli scenari, l'interconnessione dei tempi, il processo di pianificazione degli scenari e i tipi di scenario. Successivamente essi si sono cimentati con la definizione di un quadro concettuale volto a fornire le coordinate fondamentali per l'impostazione di una ricerca empirica che voglia indagare se e in che modo la metodologia dello scenario è in grado di supportare il processo di pianificazione strategica in contesti complessi. Il quadro concettuale costruito esplicita ruoli diversi in base alle diverse condizioni dell'impresa e alla complessità ambientale.

Giacomo Boesso, Fabrizio Cerbioni e Livia Mian partono dalla considerazione che la letteratura in tema di approccio strategico delle fondazioni può essere classificata in due aree. La prima tratta dell'approccio deterministico che trova attuazione quando le fondazioni sono gestite in modo manageriale. La seconda studia il ruolo solidarista e cooperativo dell'azione delle fondazioni nel sostenere la prosperità di altre organizzazioni non profit. Il lavoro proposto è fondato sui risultati di un sondaggio realizzato mediante la somministrazione di un questionario ai decisori delle principali fondazioni italiane e volto a indagare il grado di adozione dei due modelli di riferimento e la loro relazione con le prestazioni, i meccanismi di governance e il profilo strategico. I risultati mostrano che il determinismo è associato a prestazioni più elevate, mentre il solidarismo, pur ampiamente adottato, non appare in grado di generare risultati sociali positivi. Si assiste tuttavia a una progressiva contaminazione tra i due modelli.

Il lavoro di Silvia Fissi, Elena Gori, Alberto Romolini e Marco Contri conclude il fascicolo con un'indagine relativa all'utilizzo dei social media nei musei italiani. Attraverso i SM - Facebook, Twitter, Instagram e YouTube - le organizzazioni possono costruire e rafforzare le relazioni con una varietà di parti interessate. Essi consentono in particolare di realizzare una comunicazione a due vie e favoriscono il dialogo con il pubblico. Partendo 
dall'osservazione che la letteratura in tema di applicazione nel settore culturale è contenuta, gli autori analizzano l'uso dei SM da parte dei musei di stato italiani e si interrogano in particolare sul coinvolgimento dei diversi stakeholder. I risultati dell'analisi mostrano che i musei italiani utilizzano i SM con frequenza crescente; essi tuttavia non cercano di coinvolgere e impegnarsi attivamente con gli utenti, così che il loro approccio continua a essere esclusivamente comunicativo.

\section{Bibliografia}

Arena M., Arnaboldi M. (2014), Risk and performance management: are they easy partners? Management Research Review, 37, pp. 152-166. Doi: 10.1108/MRR-08-2012-0180.

Besta F. (1880), Prolusione letta nella solenne apertura degli studi per l'anno scolastico 1880-81 alla R. Scuola Superiore di Commercio in Venezia, Venezia, Italia: Tipografia dell'Istituto Colletti.

Besta F. (1922), La Ragioneria, voll. I - III, ristampa della $2^{\circ}$ edizione, riveduta e ampliata col concorso dei proff. V. Alfieri, C. Ghidiglia e P. Rigobon. Milano, Italia: Vallardi.

Cardoni A. (2018), Le sfide evolutive del management control tra relazioni strategiche, innivazione e discontinuità: a knowledge trasfer matter? Management Control, 1, pp. 5-15.

Chiucchi M.S., Gatti M. (2015), L'evoluzione degli studi di management control: un percorso nel segno della varietà, Management Control, 1, pp. 5-8. Doi: 10.3280/MACO2016002001 .

D’Onza G. (2008), Il sistema di controllo interno nella prospettiva del risk management, Giuffrè Editore, Milano

Landis C., Paglietti P. and Pavan A. (2012), I sistemi di controllo interno e la costrizione degli atti amministrativi nella scuola di Fabio Besta. Alcune riflessioni di carattere comparative, Contabilità e cultura aziendale, XII(1).

Marchi L. (2018), Quale metodologia della ricerca sulle tematiche di management control, Management Control, 2, pp. 5-10.

Marchi L. (2015), Nuove prospettive di ricerca sulle tematiche di management control, Management Control, 3, pp. 5-8. Doi: 10.3280/MACO2015-003001.

Onida P. (1965), Economia d'azienda, Torino, UTET.

Pavan A, Fadda I. (2017), Increasing the value of accounting research. An Italian perspective, Financial Reporting, 2, pp. 29-42. Doi: 10.3280/FR2017-002003.

Soin K., Collier P. (2013), Risk and risk management in management accounting and control, Management Accounting Research, 24, pp. 82-87. Doi: 10.1016/j.mar.2013.04.003.

Sòstero U. (2003), L'economicità delle aziende, Milano, Giuffrè. 